INCREASED VASOPRESSIN PRODUCTION IN SENESCENCE AND DEMENTIA DUE TO KIDNEY

CHANGES

\author{
R. Ravid, D.F. Swaab, E. Fliers and J.E. Hoogendijk \\ Netherlands Institute for Brain Research, Meibergdreef 33
}

$1105 \mathrm{AZ}$ Amsterdam Z0, the Netherlands

\title{
ABSTRACT
}

Vasopressin (VP) is involved as a neurotransmitter in a number of central functions that are frequently disturbed during aging and dementia. Therefore, this peptide has been used in clinical trials as a 'substitution therapy' for the degenerating peptidergic neurons, aimed at improving cognitive functions in aged and demented individuals with unequivocal results. In order to investigate whether the VP systems indeed show the claimed degenerative changes during aging and dementia, we focused in the first place on the Supra Optic Nucleus (SON) and the Para Ventricular Nucleus (PVN). VP cells were identified by means of immunocytochemistry in a series of 32 formalin-fixed human hypothalami, including 4 patients with senile dementia of the Alzheimer type (SDAT). In the SON and PVN, VP cell and nucleolar size was determined by means of a digitizer device, as parameter for peptide synthesizing activity. VP cell size and nucleolar size increased beyond 80 years of age, both in the PVN and in the SON. In SDAT patients these measures fell within the range for their age group. Instead of degenerative changes, these results show an activation of the vasopressinergic system, in senescence and in SDAT patients, similar to earlier observations in the aged rat and in accordance with a rise in human neurophysin and VP levels reported recently. The cause for these changes might be in the kidney.

Immunocytochemical staining of VP binding sites in the renal tubuli was strongly diminished in kidneys of old ( 25 and 34 months) as compared to young ( 3 and 5 months) Wistar and Brown-Norway rats. Urine production did not significantly increase in old age and the activation of the vasopressin neurons thus seems to be a secondary mechanism to compensate for loss of VP receptors in the old kidney.

\section{VASOPRESSIN, AGING AND DEMENTIA}

Advanced age is associated with a decreased ability of the organism to adapt to changes in its environment, and at a more fundamental level, with a decreased capacity of certain tissues to adapt their physiological responses to changes in neuronal or hormonal environment. This reduced adaptive capacity may be based upon deficiencies in neurotransmitters and neurohormones. Neuropeptides are the largest and most variable class of neurotransmitters (for review see Swaab, 1982). In the last few years, the changes 
in neuropeptide-containing neurons were studied in our group, in rat and human brain, and in patients with senile dementia of the Alzheimer type (SDAT). In the first place, the morphological basis for central effects of neuropeptides was described with special emphasis on vasopressin, which is known to be a neurohormone with many central effects (cf. De Wied, 1983). For a long time this peptide has been known to be produced in the Supra Optic Nucleus (SON) and Para Ventricular Nucleus (PVN), transported to the neurohypophysis and released as a neurohormone into the bloodstream.

The observations of De Wied et al. (1983) on vasopressin effects on 'memory' and the reports of Legros et al., (1980) on decreased plasma levels of the vasopressin precursor molecule Neurophys in in normal subjects between the ages of 55 and 60 , were a strong stimulus for the hypothesis that a degeneration of vasopressin neurons at older age would be a basis of memory impairment. This idea was reinforced by the observations of Legros et al. (1980), who reported that administration of vasopressin by nasal spray to healthy volunteers of 50-65 years of age, improved performance in psychometric tests. Later trials in aged and demented patients were no longer unequivocal, however (for review see Jolles, 1983).

\section{SON, PVN AND SUPRACHIASMATIC NUCLEUS (SCN) CHANGES DURING AGING AND DEMENTIA}

Consequently, the question was studied whether indeed the vasopressin neuron was degenerating in senescence and dementia in the rat and human brain. Combining immunocytochemical (ICC) investigations with morphometric techniques for localization of vasopressin and measuring changes in peptide production, revealed that vasopressin neurons in some brain areas indeed degenerate during aging, and even more pronouncedly in dementia (i.e., in the SCN). A marked decrease in the SCN volume, VP cell number (fig. 1) and total SCN cell number was found in 80-100 year-old patients as compared with the youngest age group (Swaab et al., 1984; Swaab et al., 1985b). However, other vasopressin neurons, viz., the SON and PVN, were found to be activated in senescence and dementia, and to maintain an increased level of neurosecretion in these conditions (Fliers and Swaab, 1983; Swaab et al., 1984; Swaab et al., 1985a,b; Fliers et al., 1985). Cell size of immunocytochemically identified VP cells was measured as a parameter for peptide production in these areas (Swaab et al., 1984; Fliers et al., 1985). A significant increase was observed in the size of VP cell profiles, beyond the age of 80 (Swaab et al., 1984; Swaab et al., 1985). In brains from patients with SDAT, this morphometric parameter was within the range of their respective age group. Assuming that the well-known relation between neurosecretory cell size and peptide-synthesizing activity (cf. Fliers et al., 1985) also holds for the aging brain, the observed increase in VP cell size after 80 years of age may imply an increased activity of these cells. This interpretation is strengthened by recent nucleolar measurements (Hoogendijk et al., 1985), which show identical results (fig. 2). Our morphometric observations are in good agreement with the decreased blood levels of neurophysins (Legros, 1975) and increased peripheral levels of VP (Frolkis et al., 1982; Kirkland et al., 1984). Legros et al. (1980) reported also a secondary increase in immunoreactive neurophysin blood levels after the age of 70. A similar activation of VP production and release was found in aged Wistar rats (Fliers and Swaab, 1983).

\section{AGE-RELATED CHANGES IN RENAL VP BINDING SITES}

Immunocytochemical staining of VP binding sites in the rat kidney has given a possible explanation for the activation of the neurosecretory function of the vasopressin neurons. This procedure revealed a significant diminution of VP binding sites in the aged kidney (Ravid et al., 1985; Swaab et al., 1985b; Swaab et al., 1985c). The staining of renal tubuli 
in young and old Wistar and Brown-Norway rats was confined to medullary and cortical portions of collecting ducts and an occasional staining in the distal convoluted tubes only after preincubation of the kidney sections with VP, which enhanced the staining. In young kidneys ( 3 and 5 months) a very intense staining of medullary collecting ducts was observed and a marked staining of cortical collecting ducts and distal convoluted tubes. In old kidneys ( 25 and 34 months) only occasional and weak staining of medullary collecting ducts was observed ( $\mathrm{fig} .3$ ). This diminution in VP binding sites in the old kidney is in accord with the impaired urinary concentrating ability (Bengele et al., 1981) and reduced c-AMP generation of the old kidney (Beck and Yu, 1982). In addition, $24 \mathrm{hr}$ VP excretion was found to be increased in the 25-month-old animals compared to the 3-monthold rats (Swaab et al., 1985b). Bengele et al. (1981) reported that the ability to conserve water in the kidney diminishes with age, and the same decrease was observed in senescent human subjects (Rowe et al., 1976). The maximum urinary concentrating ability is decreased in old subjects after VP administration and after water deprivation (Rowe et al., 1976), which suggests that the impairment is due to a decrease in renal response to VP. Bengele et al. (1981) found that the critical step for decrease in concentrating ability of old kidneys was significant impairment of water permeability across the epithelium of collecting ducts, even when normal circulating levels of VP were maintained.

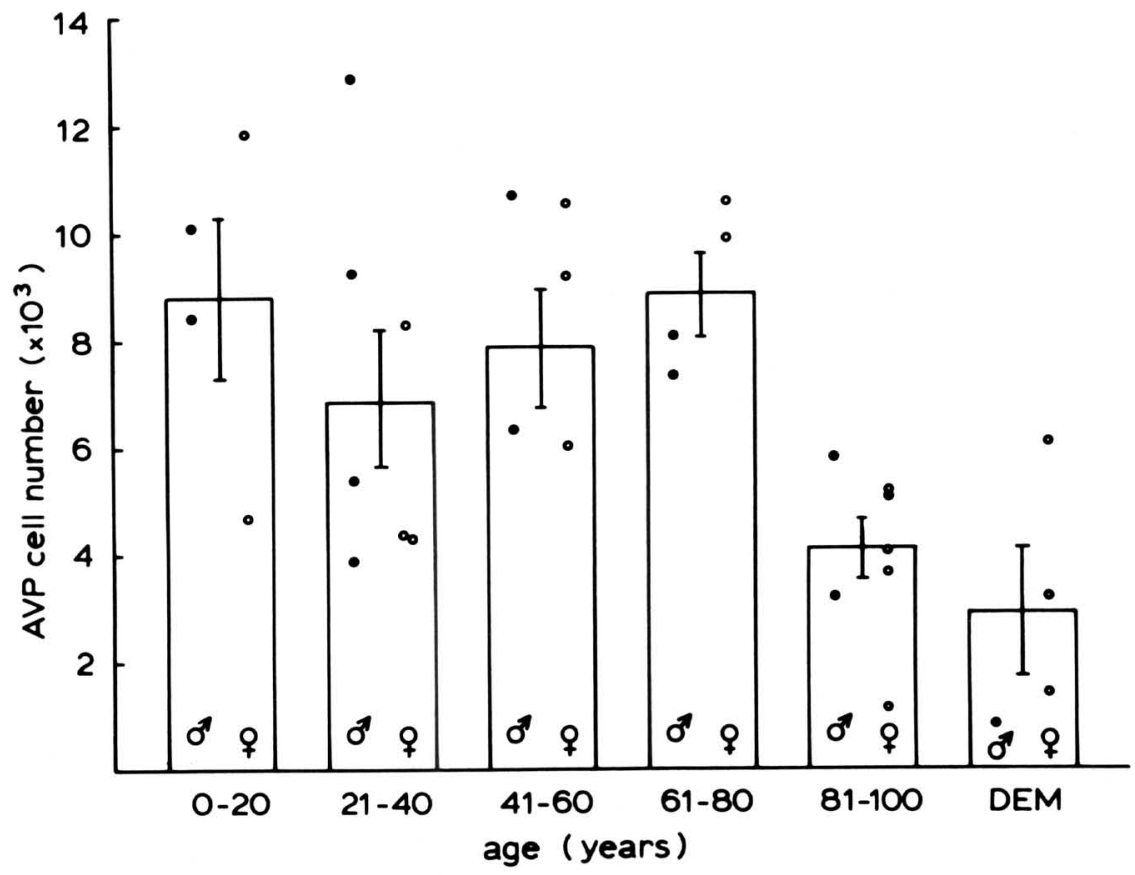

Fig. 1 - Suprachiasmatic nucleus vasopressin (AVP) cell number. Note the decrease in cell number in the oldest age group (81-100 years) and the strong decrease in the demented patients, in spite of their relatively young mean age ( 76 years). The vertical lines denote the SEM.

(This figure is reproduced by permission from the paper: The suprachiasmatic nucleus of the human brain in relation to sex, age and dementia, Brain Research, 1985, in press). 
These findings are in line with the increased activity of the vasopressin neurosecretory neuron and the enhanced VP blood levels in the old rat as described earlier (Fliers and Swaab, 1983). Urine production does not decrease at old age and the VP neuron activation in senescent rats thus seems to compensate for loss of VP receptors in the old kidney.

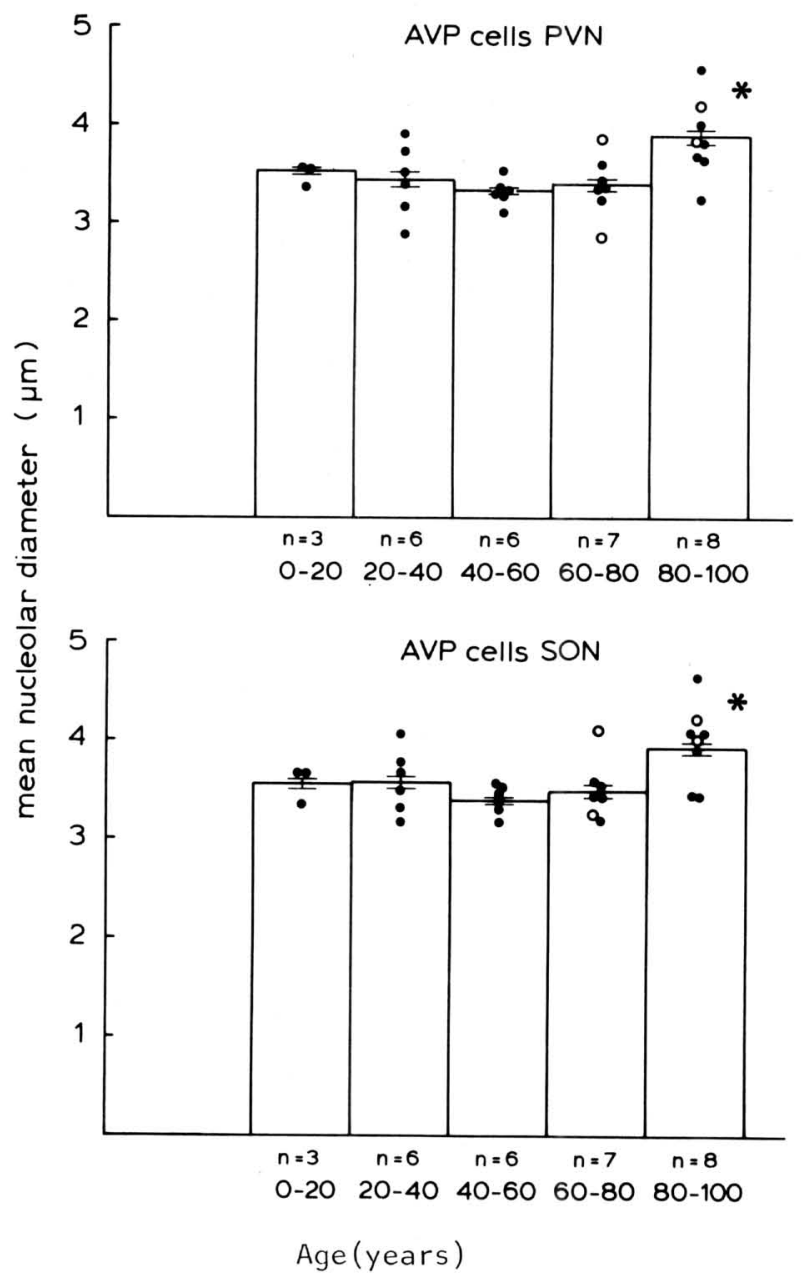

Fig. 2 - Mean nucleolar diameter of vasopressin (VP) cells in the Paraventricular Nucleus (PVN) and Supraoptic Nucleus (SON) as a function of age. There is a significant effect of age on mean nucleolar diameter of AVP cells. Values in the 80-100 year-old group are higher than the 20-80 year-old groups in the PVN and the 40-80 year-old groups in the SON, as indicated by the asterisk. Bars indicate mean values per two decades, vertical lines indicate SEM, $n$ representing the number of brains examined in each age group. Open circles represent values from SDAT patients. (This figure is reproduced by permission from the paper: Activation of the vasopressin neurons of the human supraoptic and paraventricular nucleus in senescence and senile dementia, by Hoogendijk, J.E., Fliers, E., Swaab, D.F. and Verwer, R.W.H., J. of Neurol. Sci., 1985, in press). 
3 months (a)

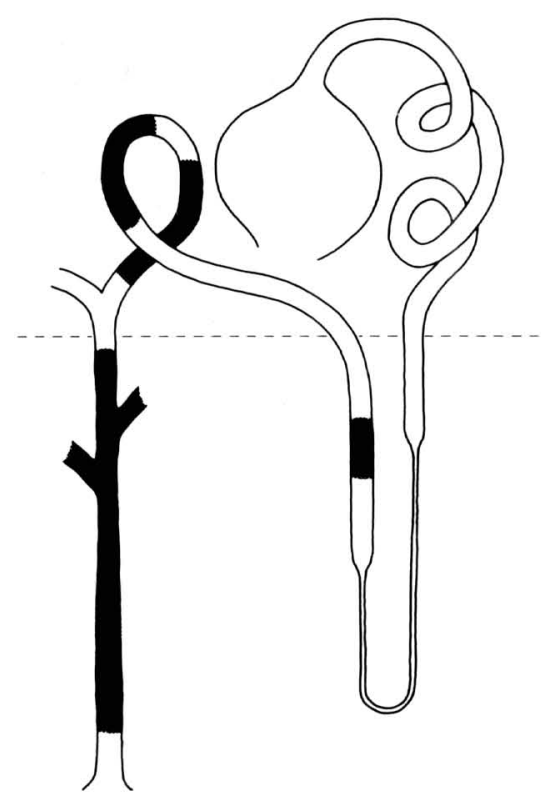

25 months (b)

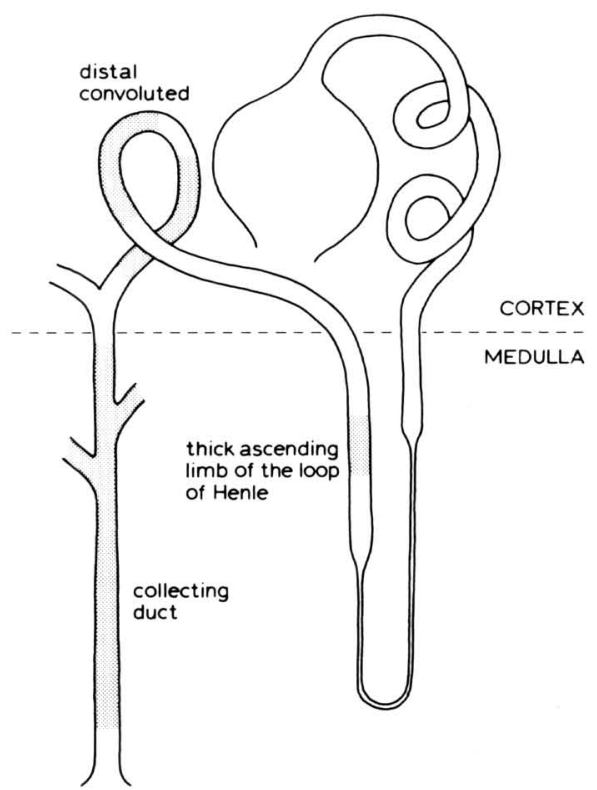

Fig. 3 - Schematic illustration of VP binding, revealed by an immunocytochemical staining method, in the kidney of Wistar rats of $3(a)$ and $25(b)$ months of age. The very intense staining of medullary collecting ducts is indicated in (a) and the weak and occasional staining of the same tubes in (b). This difference becomes most obvious when the kidney sections are incubated with 600 nM VP prior to staining with anti-VP antibody.

\section{CONCLUSIONS}

VP seems to act as a neurohormone when released from the neurohypophysis and as a neurotransmitter when released within the brain. It appears that neurosecretory VP neurons in the SON and PVN are activated in senescence, while patients with senile dementia of the Alzheimer type (SDAT) follow this curve in accordance with their age. In the SCN, on the other hand, a clear decrease in the amount of VP neurons was observed in SDAT. Depending on the area studied, the VP neuron is thus either activated or degenerated, and therefore little specific benefit might be derived from a general way of administration of exogenous VP during aging and dementia. Thus, our findings might explain the negative results of clinical trials involving VP administration to these patient groups (cf. Jolles, 1983). In addition, one should take into consideration that the primary cause of the VP neurosecretory activation seems to lie in the diminished VP binding in the kidney. Our findings also emphasize that SDAT can neither be regarded as merely a 'general degenerative process' of the brain, since VP cells in the SON and PVN are activated, nor as a 'cholinergic disease', in view of the clear changes in the SCN of VP-containing cells and the other SCN cells. These findings do not rule out beneficial effects of peptides during aging and dementia as a result of other, e.g., metabolic or trophic effects of neuropeptides or their analogues. 
Beck, N. and Yu, B.P., 1982, Effect of aging on urinary concentrating mechanism and vasopressin-dependent cAMP in rats, Am. J. Physiol., 243: F121-F125.

Bengele, H.H., Mathias, R.S., Perkins, J.H. and Alexander, E.A., 1981 , Urinary concentrating defect in the aged rat, Am. J. Physiol., 240: F147-F150.

De Wied, D., 1983, Central actions of neurohypophyseal hormones. In: The Neurohypophysis: Structure, Function and Control, Progress in Brain Research, Vol. 60, B.A. Cross and G. Leng, Eds., Elsevier, Amsterdam.

Fliers, E. and Swaab, D.F., 1983, Activation of vasopressinergic and oxytocinergic neurons during aging in the Wistar rat. Peptides, 4: 165-170.

Fliers, E., Swaab, D.F., Pool, Chr.W. and Verwer, R.W.H., 1985, The vasopressin and oxytocin neurons in the human supraoptic and paraventricular nucleus; changes with aging and senile dementia, Brain Research, in press.

Frolkis, V.V., Golovchenko, S.F., Medved, V.I. and Frolkis, R.A., 1982, Vasopressin and cardiovascular system in aging, Gerontol., 28: 290-302.

Hoogendijk, J.E., Fliers, E., Swaab, D.F. and Verwer, R.W.H., 1985, Activation of vasopressin neurons in the human supraoptic and paraventricular nucleus in senescence and senile dementia, J. of Neurol. Sci., in press.

Jolles, J., 1983, Vasopressin-like peptides and the treatment of memory disorders in man, $I n$ : The Neurohypophysis: Structure, Function and Control, Progress in Brain Research, Vol. 60, Cross, B.A. and Leng, G., Eds., Elsevier, Amsterdam, 169-182.

Kirkland, J., Lye, M., Goddard, C., Vargas, E. and Davies, I., 1984, Arginine vasopressin in dehydrated elderly patients. Clin. Endocrinol., 20: 451-456,

Legros, J.J., 1975, Radioimmunoassay of human neurophysins: contribution to the understanding of the physiopathology of neurohypophyseal function. Ann. N.Y. Acad. Sci. 248: 281-303

Legros, J.J., Gilot, P., Schmitz, S., Bruwier, M., Mantanus, H., and TimsitBerthier, M., 1980, Neurohypophyseal peptides and cognitive function: a clinical approach, In: Progress in Psychoneuroendocrinology, Brambilla, F., Racagni, G. and De Wied, D., Eds., Elsevier/North Holland Biomedical Press, 325-337.

Ravid, R., Swaab, D.F. and Pool, Chr.W., 1985, Immunocytochemical localization of vasopressin binding sites in the rat kidney. J. Endocrinol., in press.

Rowe, J.W., Shock, N.W. and De Fronzo, R.A., 1976, The influence of age on the renal response to water deprivation in man. Nephron, 17: 270-278.

Swaab, D.F., 1982, Neuropeptides. Their distribution and function in the brain. In: Chemical Transmission in the Brain. Progress in Brain Resea.rch, Vol. 55, Buijs, R.M., Pévet, P., and Swaab, D.F., Eds., Elsevier, Amsterdam, 97-122.

Swaab, D.F., Fliers, E. and Fisser, B., 1984, The vasopressin containing neuron in the human brain. Changes during aging and senile dementia. Proc. EURAGE Workshop 'Aging of the Brain and Senile Dementia: The Inventory of EEC Potentialities', D.L. Knook et al. Eds., Eurage, $71-78$.

Swaab, D.F., Fliers, E. and Partiman, T.S., 1985a, The suprachiasmatic nucleus of the human brain in relation to sex, age and dementia, Brain Research, in press.

Swaab, D.F., Fliers, E. and Ravid, R., 1985b, The vasopressin neuron in the aging humand and rat brain. In: Proceedings of the conference: 'Comparative Aspects of Opioid and Related Neuropeptides', Sunny, U.S.A., in press. 
Swaab, D.F., Fliers, E. and Van Gool, W.A., 1985c, Immunocytochemical localization of neuropeptides in the human brain; its consequences for therapeutic strategies in aging and dementia. In: Progress in Brain Research, in press.

\section{Acknowledgements}

We gratefully acknowledge the V.D. Houten Fund for supporting this research, The Dutch Gerentology Foundation for supporting the participation in the 30th Oholo Conference and Peter V. Niewkoop, G.U.D. Meulen and $H$. Stoffels for preparing the manuscript for publication. 